Pure and Applied Mathematics Quarterly

Volume 2, Number 4

(Special Issue: In honor of

Robert MacPherson, Part 2 of 3)

1237-1253, 2006

\title{
Higher Todd Classes and Holomorphic Group Actions
}

\author{
Jonathan Block and Shmuel Weinberger \\ To Robert MacPherson with admiration \\ on the occasion of his 60th birthday.
}

\begin{abstract}
This paper attempts to provide an analogue of the Novikov conjecture for algebraic (or Kähler) manifolds. Inter alia, we prove a conjecture of Rosenberg's on the birational invariance of higher Todd genera. We argue that in the algebraic geometric setting the Novikov philosophy naturally includes non-birational mappings.
\end{abstract}

\section{INTRODUCTION}

This paper describes an attempt to export the Novikov conjecture's philosophy in the direction of algebraic geometry. We hope that our discussion is useful to algebraic geometers and topologists.

Our story begins with Hirzebruch's Riemann-Roch theorem, or even earlier, with Hirzebruch's signature theorem, which was a lemma in his proof of RiemannRoch. Let $M^{4 k}$ be a smooth closed oriented manifold of dimension $4 k$. The signature of $M, \operatorname{sign}(M)$ is by definition the signature of the symmetric bilinear form

$$
\cup: H^{2 k}(M ; \mathbb{R}) \times H^{2 k}(M ; \mathbb{R}) \rightarrow H^{4 k}(M ; \mathbb{R}) \cong \mathbb{R}
$$

By Poincare duality, this is a nonsingular pairing, and since $2 k$ is even, the pairing is symmetric. Such forms can be diagonalized, and the signature is the difference in dimensions between the positive and negative definite parts.

Hirzebruch's signature theorem [27] asserts that

$$
\operatorname{sign}(M)=\langle L(M),[M]\rangle .
$$

Received November 11, 2005.

Both authors supported in part by NSF grants. The authors would like to thank Jonathan Rosenberg for enlightening discussions and comments in the preparation of this article. They would also like to thank Anatoly Libgober and Jorg Schürmann for useful correspondences and the referee for many helpful suggestions. 
where $L(M)$ is a particular graded polynomial in the Pontrjagin classes of $M$. Note that as a consequence of this, without knowing a thing more about $L$, that if $N \rightarrow M$ is a finite cover, then $\operatorname{sign}(N)=s \cdot \operatorname{sign}(M)$, where $s$ is the number of sheets of the cover. In fact, Hirzebruch was very interested in the exact formula for $L$, and it is quite intimately related to problems as disparate as the number of exotic differential structures on the sphere [30] and Euler-MacLauren formula and lattice point counting problems [14].

The two sides of the formula (1.1) are of very different sorts. The left hand side is, by definition, an oriented homotopy invariant. (It is defined cohomologically.) The right hand side seems to depend on the smooth structure. Indeed, ultimately (1.1) is one of the key ingredients in Novikov's proof that Pontrjagin classes are (rationally) topological invariants [36]. We will return to this later.

It is also quite obvious from Stokes' theorem and (1.1) that the right hand side vanishes whenever $M$ is the boundary of an oriented manifold. (If it merely bounded a chain, the cohomology class $L(M)$ might not extend). That this is true for $\operatorname{sign}(M)$ was first observed by Thom, and it is a consequence of Poincare duality for manifolds with boundary. Hirzebruch's original proof of (1.1) was a systematic exploitation of

(1) the cobordism invariance of the signature,

(2) the multiplicative formula

$$
\operatorname{sign}(M \times N)=\operatorname{sign}(M) \operatorname{sign}(N)
$$

and,

(3) Thom's calculation of oriented cobordism [46].

That $\langle L(M),[M]\rangle$ is a homotopy invariant though, seems to only follow from the signature theorem; it does not have an independent explanation.

Are there any other combinations of Pontrjagin classes that integrate to a homotopy invariant? This was considered by P.Kahn in his thesis [29], and there is no other rational homotopy invariant cobordism invariant. ${ }^{1}$

Novikov, however, suggested that if we give our manifolds "polarizations" i.e. continuous maps $f: M \rightarrow B \pi$, then we can exploit the fundamental groups to possibly obtain more homotopy invariants. (Here $B \pi$ denotes the classifying space of the group $\pi$; it is an Eilenberg space of type $K(\pi, 1)$, a space with fundamental group $\pi$ and contractible universal cover. ${ }^{2}$ )

\footnotetext{
${ }^{1}$ Note that all Stiefel-Whitney numbers are $(\bmod 2)$ invariants of homotopy type that are cobordism invariant. Indeed, it follows from the $\mathrm{Wu}$ formulae that Stiefel-Whitney classes are themselves homotopy invariant.

${ }^{2}$ Spaces with contractible universal cover are called aspherical.
} 
More precisely, Novikov conjectured that if $\alpha$ is an element of $H^{*}(B \pi ; \mathbb{Q})$ then $\left\langle f^{*}(\alpha) \cup L(M),[M]\right\rangle$ is an oriented homotopy invariant. Subsequent work of Mischenko and Wall (independently) showed that polarizations into non-aspherical spaces are useless: only cohomology classes that come from the induced map on cohomology from $M \rightarrow B \pi_{1}(M)$ have any chance of producing (polarized) homotopy invariants, and as with Kahn's theorem, there is no point in considering characteristic polynomials other than $L$.

We recommend the survey by Jim Davis [17] for more about the foundations of the Novikov conjecture. (Indeed, there are several volumes, and many surveys of this problem - we shall avoid the temptation to give a survey of these surveys, here.)

We shall return to the Novikov conjecture, but it is perhaps not to soon to expand on the Novikov philosophy in other directions.

The Riemann-Roch theorem of Hirzebruch computes the arithmetic genus $p_{a}(M)$ of a projective algebraic manifold ${ }^{3}$ in terms of another polynomial, the Todd class $\operatorname{Td}(M)$.

$$
p_{a}(M)=\langle\operatorname{Td}(M),[M]\rangle
$$

The arithmetic genus is the alternating sum of the dimensions of the Dolbeault cohomology groups of $\mathrm{M}$,

$$
\begin{aligned}
p_{a}(M) & =\sum_{i}(-1)^{i} \operatorname{dim} H^{0, i}(M) \\
& =\sum_{i}(-1)^{i} \operatorname{dim} H^{i}\left(M ; \mathcal{O}_{M}\right)
\end{aligned}
$$

The Todd class is a polynomial in the chern classes of M, and we shall, again, ignore its precise form.

Now, the analogue of the homotopy invariance consequence of (1.1) is that $\operatorname{Td}(M)$ integrates to a birational invariant of a smooth variety $M$. The reason is that the left hand side is a birational invariant; indeed, as a consequence of the Hartog extension theorem, each of the individual cohomology groups arising in the definition of the arithmetic genus are birational invariants (see [22]).

Then the Novikov philosophy of trying to couple with group cohomology to extend a general result to a more precise one in the presence of a fundamental group leads one (and in particular Rosenberg $[37]^{4}$ ) to conjecture:

\footnotetext{
${ }^{3}$ Of course, the index theoretic proof [4] of the Riemann-Roch formula removes algebraic hypotheses on $\mathrm{M}$. We have also ignored, for now, the extension to $\chi(M, V)$ where $V$ is a holomorphic vector bundle over $\mathrm{M}$.

${ }^{4}$ Actually, we had made the same conjecture some years ago, and verified in the case of abelian fundametantal group using ideas of Lusztig [33], but then noticed the more general
} 
Conjecture 1.1. (Birational invariance of higher Todd Genera) If $M$ is a smooth projective variety and $f: M \rightarrow B \pi$ is a continuous map and if $\alpha$ is an element of $H^{*}(B \pi ; \mathbb{Q})$ then

is a birational invariant.

$$
\left\langle f^{*}(\alpha) \cup T d(M),[M]\right\rangle
$$

Note (see [22] or [37]) that the (topological) fundamental group is a birational invariant, so the question makes sense. Rosenberg in fact showed that for many $\pi$, this is true; it's true whenever a certain approach to the Novikov conjecture (the so called "analytic approach") works. We will see that it's true in general using resolution of singularities and the Riemann-Roch theorem of Baum-FultonMacPherson $[6]^{5}$, but again, we are rushing the story. Rosenberg also observed an analogue of the theorems of Kahn-Mischenko-Wall, that no other combinations of Chern numbers can be birationally invariant.

This philosophy has had another very notable success, in differential geometry, regarding the problem of constructing complete metrics of positive scalar curvature. In that case, the "general theorem" is due to Lichnerowicz, as an early consequence of the Atiyah-Singer index theorem for the Dirac operator (which also implies Hirzebruch's theorems) [4], and asserts:

Theorem 1.2. (Lichnerowicz's theorem) If $M$ is a spin manifold with a metric of positive scalar curvature, then $\langle\hat{A}(M),[M]\rangle=0$, where $\hat{A}(M)$ is the $\hat{A}$-genus.

And again, there is the:

Conjecture 1.3. (Gromov-Lawson-Rosenberg) If $M$ is a spin manifold with positive scalar curvature and and $f: M \rightarrow B \pi$ is a continuous map and if $\alpha$ is an element of $H^{*}(B \pi ; \mathbb{Q})$ then $\left\langle f^{*}(\alpha) \cup \hat{A}(M),[M]\right\rangle=0$.

The analogy between this problem and the Novikov conjecture was developed in Rosenberg, [38]. Moreover, see [44] for an explanation of the "converse theorems", based on a surgery theorem of Gromov-Lawson and Schoen-Yau, and spin cobordism calculations of Stolz, as well as many more positive results.

Having mentioned the Atiyah-Singer theorem and elliptic operators, it is now inevitable that we bring in K-theory . (The Grothendieck part of the story will come in the next section.) In the original papers of Atiyah and Singer, they associated a "symbol bundle" to any elliptic operator 6 [D] in $K^{*}\left(T^{*} M\right)$, where

results described below so we let the matter of finding an analogue of the Novikov conjecture drop. On reading [37], we decided to return to the problem.

${ }^{5}$ This follows from the preprint [13] as well. Moreover, the precise integral statement that for smooth birational morphisms the derived pushforward of the structure sheaf is the structure sheaf, which is the key point in our proof, also follows from [45]

${ }^{6}$ We do not distinguish between an elliptic operator and an elliptic complex, nor between a sheaf or a complex of sheaves. 
$T^{*} M$ is the cotangent bundle of $M$. (For us $K^{*}$ denotes the topological $K$ group.) However, $T^{*} M$ has a natural symplectic structure, $\omega$ hence is orientable for K-theory, and we can thus associate to $D$ an element of the dual homology theory $K_{*}(M)$. The index theorem then asserts that $\operatorname{ind}(D)=\operatorname{dim} \operatorname{ker}(D)-$ $\operatorname{dim} \operatorname{cok}(D)=p_{*}[D]$ in $\mathbb{Z}$, where $p$ is the constant map from $M \rightarrow$ pt a point. We can also explain the index theorem for families easily in this framework, but we shall not. (We might suggest that the reader consult [2] for an early approach to the K-homology class associated to an elliptic operator, and [26] for a recent text.)

In all the above examples there are operators, namely the "signature", Dolbeault, and Dirac operators, which give "symbol classes" in $K_{*}(M)$. Now, instead of considering $p: M \rightarrow \mathrm{pt}$, we consider $f: M \rightarrow B \pi$. This then gives us $f_{*}[D] \in K_{*}(B \pi)$ and one can conjecture appropriate vanishing or invariance properties of this invariant. We call this the integral Novikov conjecture.

By using the Chern character, rational K-homology is identified with ordinary rational homology, and the conjectures discussed above are the vanishing of this homology class by checking that its pairing with arbitrary cohomology classes vanishes.

Moreover, this inclusion of torsion is extremely significant. For instance, if one uses real K-theory, then $K O_{*}(\mathrm{pt})$ has 2-torsion, and one obtains a more general obstruction to positive scalar curvature [28], which can be used to show that certain homotopy spheres do not have positive scalar curvature.

Nice as all of this is, it's off in detail. The integral Novikov conjecture, as we just stated it, is wrong for signature and Dirac. In both cases, it's the same counterexample. If one considers lens spaces of high dimensions with fundamental group $\mathbb{Z} / p, p$ a large prime, it is easy to do calculations to give the non-homotopy invariance of the K-theoretic signature class. (In defining lens spaces, one takes the quotient of a sphere under a free linear action of a cyclic group: by varying the linear representation of $\mathbb{Z} / p$, one gets many examples of nondiffeomorphic, but homotopy equivalent manifolds, see [35].) Similarly, all lens spaces have positive scalar curvature, but the Dirac class is nontrivial. These classes are all torsion, though, so this issue does not affect the (rational) Novikov conjecture.

Now, there is a very sensible way to formulate an integral Novikov conjecture, even in the presence of torsion, and it boils down to what we said above for $\pi$ torsion free, but we shall not pursue it here. (See e.g. [5], [48] for some discussions: essentially one studies the invariants of proper but perhaps non-free actions on contractible spaces rather just free actions.)

In any case, the next result is not analogous to what occurs for the other operators: it is too strong. 
Proposition 1.4. The higher Todd K-class $f_{*}([$ Dolbeault $]) \in K_{*}(B \pi)$ is always a birational invariant.

In the next section we shall prove the proposition above and in the final section give what we think are examples of phenomena which more closely follow the Novikov philosophy.

\section{Novikov COnJeCtures AND Novikov theorems}

Let us recall the Grothendieck-Riemann-Roch theorem of Baum, Fulton and MacPherson, [6]. For them, the novelty was to extend the Riemann Roch theorem to singular spaces. We however only need it for smooth ones, but we like their statement because it takes values in topological $K$-theory.

Let $K_{0}^{a}(X)$ denote the Grothendieck group of coherent sheaves on the algebraic variety $X$. Grothendieck realized that taking the Euler characteristic of a coherent sheaf was a special case of pushing forward in algebraic $K$-theory. Thus the Riemann-Roch problem is about passing from the algebraic/geometric group $K_{0}^{a}(X)$ to receptacle theories which are presumably easier to compute with and also understanding how this map behaves with respect to pushforward. For example, the Todd genus is the correction needed to make the corresponding map on chow groups (or singular homology) commute, [8]. Baum, Fulton and MacPherson emphasized that there are other interesting targets, and in [6] they used topological $K$-homology.

Recall how the pushforward in algebraic $K$-theory works. Given a proper map $f: X \rightarrow Y$ between algebraic varieties, one defines

$$
f_{*}: K_{0}^{a}(X) \rightarrow K_{0}^{a}(Y)
$$

for $A$ a coherent sheaf on $X$ and $[A]$ its class in $K_{0}^{a}$

$$
f_{*}[A]=\sum_{i}(-1)^{i}\left[R^{i} f_{*}(A)\right]
$$

where $R^{i} f_{*}(A)$ denotes the $i$ th higher pushforward. This is the sheafification of

$$
U \mapsto H^{i}\left(f^{-1}(U) ; A\right)
$$

It is true that $R^{i} f_{*}(A)$ is a coherent sheaf and that the class of $f_{*}(A)$ only depends on the class of $[A]$.

To be a suitable receiver, the theory needs to have pushforwards for proper maps, so that one can compare them with the pushforwards in algebraic $K$ theory. In topological $K$ theory, the pushforward is defined using duality and Gysin maps, [6].

We now recall 
Theorem 2.1. (Baum, Fulton, MacPherson) In the category of quasi-projective schemes over $\mathbb{C}$, there is a natural transformation

$$
\alpha: K_{0}^{a}(X) \rightarrow K_{0}(X)
$$

Furthermore, $\alpha$ is commutes with proper pushforwards.

Now for a complete variety $X$ the pushforward of $\left[\mathcal{O}_{X}\right] \in K_{0}^{a}$ to a point is the arithmetic genus. On the other hand, we can take $\alpha\left(\left[\mathcal{O}_{X}\right]\right) \in K_{0}(X)$ and then pushforward. This is now some topologically computed number that equals the arithmetic genus, by the Riemann Roch theorem. The Todd genus shows up when one further takes the Chern character map from $K_{0}(X) \rightarrow H_{\text {even }}(X ; \mathbb{Q})$.

Some of the pleasing aspects of the $K_{0}$-valued Riemann-Roch theorem are

(1) The Riemann-Roch map is quite easy to define and after the relevant dualities boils down to the forgetful map on the cohomological $K$-theories:

$$
K_{a}^{0}(X) \rightarrow K^{0}(X)
$$

where $K_{a}^{0}(X)$ is the Grothendieck group of algebraic vector bundles on $X$ and $K^{0}(X)$ is the $K$-group of topological vector bundles on $X$ and the map just forgets the algebraic structure.

(2) There is no correction term since they are both forms of $K$-theory.

(3) $K_{0}(X)$ can capture torsion information.

We note the following example of pushforward as a rather simple lemma. (This appears as example 3, page 102 of [8].)

Lemma 2.2. Let $f: X \rightarrow Y$ be a morphism induced from blowing $Y$ up along a smooth center. That is $f$ is a blow down morphism. Then

$$
\begin{array}{r}
R^{0} f_{*}\left(\mathcal{O}_{X}\right)=\mathcal{O}_{Y} \\
R^{i} f_{*}\left(\mathcal{O}_{X}\right)=0 \text { for } i \neq 0
\end{array}
$$

That is,

$$
f_{*}\left(\left[\mathcal{O}_{X}\right]\right)=\left[\mathcal{O}_{Y}\right]
$$

in $K_{0}^{a}(Y)$.

Proof. This follows merely from the fact that the fibers of a blow up over a smooth center are either points or $\mathbb{P}^{n}$ 's and that in both cases $H^{i}(-, \mathcal{O})=\mathbb{C}$ if $i=0$ and 0 otherwise.

Proof. We now prove Proposition 1.4. Like Rosenberg, [37] we will use the weak factorization theorem [1]. 
Theorem 2.3. Let $\varphi: X \rightarrow Y$ be a birational map between complete nonsingular algebraic varieties over an algebraically closed field of characteristic 0. Let $U$ be an open set where $\varphi$ is an isomorphism. Then $\varphi$ can be factored into a sequence of birational maps

$$
\underset{\varphi_{1}}{\rightarrow} X_{1} \stackrel{\varphi_{2}}{\rightarrow} X_{2} \stackrel{\varphi_{3}}{\rightarrow} \cdots \stackrel{\varphi_{k-1}}{\rightarrow} X_{k-1} \stackrel{\varphi_{k}}{\rightarrow} X_{k}=Y
$$

where

(1) $\varphi=\varphi_{k} \circ \varphi_{k-1} \circ \cdots \circ \varphi_{1}$

(2) $\varphi_{i}$ are isomorphisms on $U$

(3) either $\varphi_{i}: X_{i-1} \rightarrow X_{i}$ or $\varphi_{i}^{-1}: X_{i} \rightarrow X_{i-1}$ is a morphism of algebraic varieties (in particular, everywhere defined) obtained by blowing up a smooth irreducible center disjoint from $U$.

Given a birational map $\varphi: X \rightarrow Y$, it induces an isomorphism of fundamental groups

$$
\varphi: \pi_{1}(X) \rightarrow \pi_{1}(Y)
$$

which we denote simply by $\pi_{1}$. Choosing a polarization (in the sense above), $\rho: Y \rightarrow B \pi_{1}$ induces a polarization for $X$. Now we apply the weak factorization to the birational map $\varphi$ to factor it as in (2.2). All the spaces $X_{i}$ thus inherit factorizations $\rho_{i}: X_{i} \rightarrow B \pi_{1}$ making all the maps to $B \pi_{1}$ commute. Thus, in order to prove the proposition it only remains to show that given a commutative diagram

$$
\begin{array}{r}
V \stackrel{\psi}{\rightarrow} W \\
\rho_{V} \searrow \downarrow \rho_{W} \\
B \pi_{1}
\end{array}
$$

where $\psi$ is a blowdown morphism along a smooth center, that

$$
\rho_{V}\left(\alpha_{V}\left(\mathcal{O}_{V}\right)\right)=\rho_{W}\left(\alpha_{W}\left(\mathcal{O}_{W}\right)\right) .
$$

Consider the commutative diagram

$$
\begin{aligned}
& K_{0}^{a}(V) \stackrel{\alpha_{V}}{\rightarrow} K_{0}(V) \searrow \\
& \psi_{*} \downarrow \\
& K_{0}^{a}(W) \stackrel{\alpha_{W}}{\rightarrow} K_{0}(W) \nearrow
\end{aligned}
$$

According to the lemma above, we know that

$$
\psi_{*}\left(\left[\mathcal{O}_{V}\right)\right]=\left[\mathcal{O}_{W}\right] \in K_{0}^{a}(W)
$$

So we have

$$
\rho_{W}\left(\alpha_{W}\left(\mathcal{O}_{W}\right)\right)=\rho_{W}\left(\alpha_{W}\left(\psi_{*}\left(\mathcal{O}_{V}\right)\right)\right)
$$

But by the Riemann-Roch Theorem this is

$$
\rho_{W}\left(\psi_{*}\left(\alpha_{V}\left(\mathcal{O}_{V}\right)\right)\right) .
$$


Now since $\rho_{W} \circ \psi$ is homotopic to $\rho_{V}$, we have that

$$
\left.\rho_{W}\left(\psi_{*}\left(\alpha_{V}\left(\mathcal{O}_{V}\right)\right)\right)=\rho_{V}\left(\alpha_{V}\left(\mathcal{O}_{V}\right)\right)\right)
$$

which finishes the proof.

If we examine the proof given above we now see why the birational invariance of the higher Todd class is true generally: it is because birational equivalence is hereditary, that is, it is a condition that is locally checkable on the image. As such, it is more closely analogous to Novikov's theorem that rational Pontrjagin classes are topologically invariant than it is to the Novikov conjecture ${ }^{7}$.

Let us amplify this point. If one has a homotopy equivalence $h: M^{\prime} \rightarrow M$, then one does not at all know that $h$ restricts nicely to $h^{-1}(U)$ for subsets $U \subset M$. The Novikov conjecture actually addresses this. If $f: M \rightarrow S^{1}$ is a map, and if one is able to homotop $h$ so that $h$ restricted to $h^{-1}(N)$, for $N=f^{-1}(1)$, is a homotopy equivalence, then by the Hirzebruch signature theorem, one would have obtained a proof for the fundamental class of the circle. (A sort of converse to this argument can be given via surgery theory.) In fact, this is essentially the method used in [19] in the first proof for free abelian groups. However, it is deep, and requires a homotopy to see any hereditary aspect.

However, homeomorphisms don't present this problem: they are hereditary homotopy equivalences! In fact for all open sets, they are proper homotopy equivalences. (In fact, they are bounded homotopy equivalences on all open subsets, when remetrized to be complete.) This is what leads to Novikov's theorem.

In fact, Sullivan realized that all that Novikov used was the hereditary homotopy equivalence property, and as such, Novikov's argument applies to CE maps, i.e. maps with (Cech) contractible point inverses. This doesn't give much more, though, because Siebenmann [41] showed that all such maps are uniform limits of homeomorphisms, but it does gain punch if one realizes that as rational homology is all that's ever used, one gets the same conclusion if the map were $\mathbb{Q}$-CE, i.e. had (Cech) rationally acyclic point inverse images.

Again the exact same reasoning shows that if $h: X \rightarrow Y$ is a small resolution (see [21]) then $h_{*}(L(X))=L(Y)$ where $L$ here is the Goresky-MacPherson $L$ homology class of a (suitable) stratified space. It is true on the characteristic class level, because an appropriate statement is true on the sheaf level. This same line of thought can lead one to the projection formulae in [15], which if rephrased purely homologically (rather than geometrically, as something about "stratified maps") can be held to include Novikov's theorem.

\footnotetext{
${ }^{7}$ See $[20],[16]$ for a discussion of the Novikov conjecture and Novikov philosophy on noncompact manifolds (as influenced most directly by Roe and Higson); in particular the first reference explains how to prove Novikov's theorem as a consequence of a the Novikov conjecture for the metric manifold $\mathbb{R}^{n}$.
} 
As a perhaps surprising negative example, positive scalar curvature should not be thought of local! Certainly there is no local connection between the p.s.c. assumption and characteristic classes: [31] show that any function $\phi: M \rightarrow \mathbb{R}$, which is negative somewhere, e.g. in a little ball, is the scalar curvature of a metric on a compact manifold $M$. The negative scalar curvature set can be tiny even if there's a "big" cohomological obstruction to positive scalar curvature ${ }^{8}$.

Moreover, in truth, one cannot get any information from incomplete metrics and the restriction of a given metric to an open subset will be incomplete. Any manifold has an open subset diffeomorphic to $T^{n-1} \times \mathbb{R}$, which does not have any complete p.s.c. metric - so there is no hope of a deformation argument. In the end, the p.s.c. condition is more like a global hypothesis than a local one!

\section{Connections to Group Actions}

In this section we review some connections between the Novikov philosophy and group actions, and use this to suggest a holomorphic problem which does seem tied to the Novikov philosophy. We shall also point out how the known "universal results", which apply to all fundamental groups, are essentially exploitations of the hereditary nature of hypotheses, and so tend to be correct for connected groups, but unavailable for disconnected ones.

Our story here starts off with another theorem about vanishing of the $\hat{A}$-genus of spin manifolds, here in the presence of a circle action.

Theorem 3.1. (Atiyah-Hirzebruch [3]) If $M$ is a spin manifold admitting a (nontrivial!) smooth circle action, then $\langle\hat{A}(M),[M]\rangle=0$.

There is actually a slight connection to Lichnerowicz's result above. If one had a compact nonabelian group action, rather than a circle action, then one can produce [32] an invariant positive scalar curvature metric (essentially by making the orbits have very small diameter). So, in that case one gets the Atiyah-Hirzebruch vanishing from the Lichnerowicz. However, as there are many manifolds (e.g. tori) with circle actions and no p.s.c. metrics, the results are quite independent.

It is worth noting, moreover, that the above Atiyah-Hirzebruch theorem fails for the torsion part of the index of the Dirac operator. It is tied just to the rational part. (Conversely, one does obtains a deep interesting restriction on the manifolds admitting smooth nonabelian connected Lie group actions from the [32] construction combined with Hitchin's refinement, [28] that does not seem to have a purely differential topological proof.)

${ }^{8}$ This is not the case in the noncompact case, see [40], [7]. 
One cannot go too far in guessing a nonsimply connected version of the above, as the torus has a free circle action, but its higher signature (associated to the fundamental class) is nontrivial. The way around this is to note that if a circle acts on any space $X$, the orbit of the base point defines a loop, whose class $\langle$ orbit $\rangle \in \pi_{1}(X)$ is actually central in the fundamental group. (It is part of the induced map on $\pi_{1}$ by the map $S^{1} \times X \rightarrow X$ defining the action!) It thus makes sense to work with the quotient group $\pi_{1}(M) /\langle$ orbit $\rangle$.

The following theorem was conjectured by Reinhard Schultz, motivated by the Novikov and Gromov-Lawson-Rosenberg conjectures.

Theorem 3.2. (Browder-Hsiang [12]) If $M$ is a spin manifold, $S^{1}$ acts nontrivially on $M$, and $f: M \rightarrow B \pi$ classifies the fundamental group of $M$, then for any $\alpha \in H^{*}\left(B(\pi /\langle\right.$ orbit $\rangle ; \mathbb{Q})$ one has $\left\langle f^{*}(\alpha) \cup \hat{A}(M),[M]\right\rangle=0$.

The basic idea of their proof is this: essentially ${ }^{9}$ they build an equivariant map from $M \rightarrow B \pi /\langle$ orbit $\rangle$ (where that latter has a trivial action). If one believes this, then theorem follows. Without loss of generality we can think of $B \pi /\langle$ orbit $\rangle$ as a manifold, and a cohomology class on it as being dual to a submanifold with stably trivial normal bundle ${ }^{10}$. The transverse inverse image of this submanifold has $\hat{A}$ genus exactly equal to the associated higher $\hat{A}$-genus of $M$, but this submanifold is both spin and invariant under the circle action and hence has vanishing $\hat{A}$-genus.

The proof is thus a perfect example of the locality (not in $M$, but in the target space $B(\pi /\langle$ orbit $\rangle)$. Somewhat related, and simpler, is the following result (which does have some torsion information, but which we shall not explain):

Theorem 3.3. ([49]) If $M$ is an oriented manifold ${ }^{11}$ which admits a nontrivial $S^{1}$ action with nonempty fixed point set $F$, then there is a natural orientation on $F$ and the higher signatures of $M$ and $F$ agree in $H_{*}(B \pi ; \mathbb{Q})$.

Note that if $F$ is nonempty, $\langle$ orbit $\rangle$ is trivial. The proof is similarly local over the quotient starting with a general result: The signature of the manifold equals the signature of its fixed point set. This follows from the $G$-signature theorem.

Irrelevant but irresistible (to us) remark ${ }^{12}$ : This principle is very useful for calculations. Here is a particularly amusing example. Consider a toric surface

\footnotetext{
${ }^{9}$ We are oversimplifying for convenience of exposition.

${ }^{10}$ Note that we are working rationally. The conventional argument assumes that the cohomology class is odd dimensional, and one then finds a submanifold with trivial normal bundle using old results of Serre [42]. Then one uses tricks to reduce to this, because for even dimensional classes cup square obstructs finding a dual class with trivial normal bundle. However, even in the even case, there is a dual submanifold whose normal bundle is stably trivial, and whose Euler class exactly accounts for the cup square.

${ }^{11}$ Rational homology manifold actually suffices.

${ }^{12}$ This was observed by Cappell and the second author many years ago as part of some unwritten joint work.
} 
whose moment map has image a triangle in the plane. This manifold has many different circle actions, as a torus contains many different circles. Ignoring the three circles that define the sides of the triangle, all circles have the same three fixed points $=$ the vertices of the moment polygon. The sign of the fixed point is determined by whether the line lies within the angle at the corresponding fixed point a.k.a. vertex. Considering first a line at a tiny angle with one side and then applying the observation that signature must be independent of this, we see that (almost) every line is actually in exactly one vertex angle. This is Euclid's theorem that the sum of the angles of a triangle is 180 degrees, but phrased more to his liking; the sum of the angles of a triangle is a straight angle.

However, the main point of [49] and more relevant to us is that there are versions of the above theorem for certain finite cyclic group actions that are equivalent to the Novikov conjecture.

Theorem 3.4. Suppose $G$ is a finite cyclic group which acts smoothly and twistedhomologically trivially on a smooth manifold $M$. Then the following formula for characteristic classes

$$
f_{*}(L(M) \cap[M])=(f i)_{*}\left(\left(L(F) \cup k\left(\nu_{F}\right)\right) \cap[F]\right) \in H_{*}\left(B \pi_{1}(M)\right)
$$

holds if the Novikov conjecture holds for $\pi_{1}(M)$. Here $k$ is a certain characteristic class (discussed below), $\nu_{F}$ denotes the equivariant normal to $F$ and $i: F \rightarrow M$ is the inclusion map. Conversely, if this formula holds for all such actions of a specific finite cyclic group, and even only for free actions, one can deduce the Novikov conjecture for $\pi_{1} M$.

We shall describe the characteristic class in terms that makes its version for other elliptic operators transparent. If $D$ is an elliptic operator, and $g$ is a self map of $M$ which preserves $D$, then there is a Lefshetz version of $\operatorname{ind}(D)$. One considers $L(g, D)=\operatorname{tr}(g \mid \operatorname{ker} D)-\operatorname{tr}(g \mid \operatorname{cok} D)$.

If $g$ is part of a compact group, then Atiyah and Singer give a characteristic class formula. $L(g, D)$ is the result of integrating a characteristic class over $F$, the fixed set of $g$. For us, $k$ is the result of averaging this local class for all of the generators of the cyclic group generated by $\langle g\rangle$. (They all have fixed set $F$, of course.)

Now let us be a little more explicit about the homological triviality condition. Firstly we assume that the $G$ action lifts to the universal cover as part of a $G \times \pi$ action. (This is like what happens when one has a circle action with nontrivial fixed point set.) Then we can consider the action on the homology of the universal cover or its compactly supported cohomology or its cohomology, it doesn't much matter. We assume this action is trivial.

That this formula should hold under a suitable Novikov conjecture hypothesis is most easily seen using the ideas of [39], which gives a Lefshetz point of view 
on the analytic Novikov ideas. We should therefore be a bit more precise about a formal aspect of the latter now,

At the core of this approach is the theory of $C^{*}$-algebras. It turns out that when a an elliptic operator acts on sections of a bundle whose fibers are finitely generated projective modules over a $C^{*}$-algebra $A$, its kernel and cokernel (after perturbation) can be thought of as projective modules over that algebra. Thus one has an index which lies in $K_{0}(A)$. For purposes of the Novikov conjecture the relevant algebra $C^{*} \pi$ is a completion of the integral group ring. One can take an operator on $M$, and pass to the universal cover and keep track of the $\pi$ action. Equivalently, one is taking coefficients in the tautological $C^{*} \pi$-bundle over $M$ and taking its index.

In any case, one is lead to study a natural map $K_{*}(B \pi) \rightarrow K_{*}\left(C^{*} \pi\right)$ which takes the higher symbol index to the difference between a kernel and a cokernel, i.e. a real live analytic index. If this map is injective, then we say that the analytic Novikov conjecture holds, and when the latter holds, one can deduce the ordinary Novikov conjecture and the Gromov-Lawson-Rosenberg conjecture.

The homological triviality implies that $g$ acts trivially on suitable modules, so $t r=$ dimension. So ind $(D)$, which includes the higher signature, is related to $L(h, D)$ for each $h$ which generates $\langle g\rangle$. We thus get many formulae for $\operatorname{ind}(D)$, whose average is the one displayed.

The converse result relies on surgery ideas to construct enough actions to contradict the formula if the Novikov conjecture fails. One also needs to give a purely surgery theoretic proof of the formula on the assumption of the original Novikov conjecture rather than the analytic one, which is why one needs exactly the class $k$ rather than any of its non-Galois invariant versions.

Note that this Lefshetz type localization formula is equivalent to the Novikov conjecture and thus is at least at this time not something universally true. It also doesn't hold integrally (in general). The point is that its hypothesis is not at all local. The homological triviality hypothesis is global. When one has a local reason for homological triviality, then one can indeed prove a suitable vanishing theorem. For instance if $M$ had an equivariant map to $B \pi$ (trivial action) so that all point inverses were acted on homologically trivially, then indeed one can directly prove that the higher signatures of $M$ vanish. (See [50] for related statements.)

However, we can now state and sketch a holomorphic statement which is also non-local, and which follows from the Novikov conjecture.

Theorem 3.5. Suppose that $M$ is a smooth compact Kähler manifold and $G$ is a cyclic group acting holomorphically on $M$ with fixed set $F$ and trivially on the unreduced holomorphic $L^{2}$ cohomology of its universal cover. If the analytic 
Novikov conjecture for $\pi_{1}(M)$ holds, then

$$
f_{*}(T d(M) \cap[M])=f i_{*}\left(\left(T d(F) \cup k^{\prime}\left(\nu_{F}\right)\right) \cap[F]\right) \in H_{*}\left(B \pi_{1} M\right)
$$

for a suitable characteristic class $k^{\prime}$ of the equivariant normal bundle of $F$.

The hypothesis again demands a lift of $G \times \pi$ to the universal cover of $M$. The relevant $L^{2}$ cohomology is the unreduced one (see e.g. [18]) By holomorphic part, we mean the $0, *$ part in the Hodge decomposition. The condition of homological triviality means that if we take the differential forms and compress by the idempotent

$$
p=1-\frac{1}{|G|} \sum g
$$

the spectrum of the Laplacian does not contain 0 . This implies the vanishing of the relevant Lefschetz number is equal to the index of the Dolbeault.

It is worth noting that the topological hypothesis in Theorem 3.4 suffices, in light of Hodge theory to imply the hypothesis of this theorem. (See [23], [43] for some discussion of Hodge theory on the universal covers of Kähler manifolds.) The reason is this: If the cohomology of the universal cover is a trivial $G$-module when contracted by the idempotent $p$, then the chain complex is acyclic. Since $L^{2}(\pi)$ is a module over $\mathbb{Q} \pi$, a fortiori that cohomology, when contracted by $p$ vanishes as well, i.e., the hypothesis of Theorem 3.5 holds as well. Consult [18] or [34].

Note that the above argument shows that all the cohomology groups vanish when contracted by $p$, and that therefore all the diagonals in the hodge diamond have vanishing Euler characteristic. These diagonals are Dolbeault cohomology with coefficients in a bundle, and hence, these are indices as well: the Hirzebruch $\chi_{y}$-genus encodes all of these. His theorem (which for varieties unifies Riemann Roch and the signature theorem) asserts that

$$
\chi_{y}(M)=\left\langle T_{y}(M),[M]\right\rangle
$$

for a suitable characteristic class. Combining all of the above we obtain:

Theorem 3.6. Suppose that $M$ is a smooth algebraic variety and $G$ is a cyclic group acting holomorphically on $M$ with fixed set $F$ and trivially on the cohomology with compact supports of its universal cover, then if the analytic Novikov conjecture for $\pi_{1} M$ holds, then

$$
f_{*}\left(T_{y}(M) \cap[M]\right)=f i_{*}\left(\left(T_{y}(F) \cup k_{y}^{\prime}\left(\nu_{F}\right)\right) \cap[F]\right) \in H_{*}\left(B \pi_{1} M\right)
$$

for a suitable characteristic class $k_{y}^{\prime}$ of the equivariant normal bundle of $F$ (produced from the Atiyah-Singer integrand by the usual averaging procedure).

We close with a number of problems. 
(1) The results about circle actions held for both the $L$-genus and the $\hat{A}$ genus. Presumably there is some simultaneous generalization that applies to higher elliptic genera. Some useful references that discuss elliptic genera in the context of birational geometry are [47], [9], [10],[11],[13], [45].

(2) Are there examples that show that our vanishing theorem for higher Todd genera are false for finite $\pi$, if one does not rationalize?

(3) If $f: M^{\prime} \rightarrow M$ is a holomorphic map which induces an isomorphism on $H_{*}$ for $\pi$ covers, do they have the same higher $T_{y}$-genus in $H_{*}(B \pi)$ ? For the ordinary Novikov conjecture, such a generalization is possible, and is part of the proof of the higher Lefshetz localization theorem.

(4) Is there a version of Nielsen theory for elliptic operators other than De Rham?

\section{REFERENCES}

[1] D. Abramovich, K. Karu, K. Matsuki, J. Włodarczyk, Torification and factorization of birational maps, J. Amer. Math. Soc., vol. 15, 2002, No. 3, 531-572.

[2] M. Atiyah, Global theory of elliptic operators. 1970 Proc. Internat. Conf. on Functional Analysis and Related Topics (Tokyo, 1969) pp. 21-30 Univ. of Tokyo Press, Tokyo

[3] M.Atiyah and F.Hirzebruch, Spin-manifolds and group actions. 1970 Essays on Topology and Related Topics (Mémoires dédiés a Georges de Rham) pp. 18-28 Springer, New York

[4] M.Atiyah and I.Singer, The index of elliptic operators. III. Ann. of Math. (2) 871968 $546-604$

[5] P.Baum and A.Connes, Geometric $K$-theory for Lie groups and foliations. Enseign. Math. (2) 46 (2000), no. 1-2, 3-42.

[6] P.Baum, W.Fulton, and R.MacPherson, Riemann-Roch and topological $K$ theory for singular varieties. Acta Math. 143 (1979), no. 3-4, 155-192.

[7] J.Block and S.Weinberger, Large scale homology theories and geometry. Geometric topology (Athens, GA, 1993), 522-569, AMS/IP Stud. Adv. Math., 2.1, Amer. Math. Soc., Providence, RI, 1997.

[8] A.Borel and J.P.Serre, Le théorème de Riemann-Roch. (French) Bull. Soc. Math. France 861958 97-136.

[9] L. Borisov, A. Libgober, Elliptic genera of singular varieties, Duke Math. J.,116,2003, 319351.

[10] L. Borisov, A. Libgober, Elliptic genera of singular varieties, orbifold elliptic genus and chiral de Rham complex, em Mirror symmetry, IV , 33, 325-342, 2002.

[11] L. Borisov, A. Libgober, Elliptic genera of toric varieties and applications to mirror symmetry, Invent. Math., 140, 2000, 453-485.

[12] W. Browder, W. C. Hsiang, $G$-actions and the fundamental group, Invent. Math. 65 (1981/82), no. 3, 411-424.

[13] P.Brasselet, J. Schuermann, and S.Yokura, Hirzebruch classes and motivic Chern classes for singular spaces. math.AG/0503492

[14] S.Cappell and J.Shaneson, Euler-Maclaurin expansions for lattices above dimension one. C. R. Acad. Sci. Paris Sr. I Math. 321 (1995), no. 7, 885-890.

[15] - and Stratifiable maps and topological invariants. J. Amer. Math. Soc. 4 (1991), no. $3,521-551$. 
[16] S.Chang and S.Weinberger, On Novikov type conjectures, Clay Institute volume on Noncommutative geometry, ed by N.Higson, J.Roe, and J.Rosenberg, to appear.

[17] J.Davis, Manifold aspects of the Novikov conjecture. Surveys on surgery theory, Vol. 1, 195-224, Ann. of Math. Stud., 145, Princeton Univ. Press, Princeton, NJ, 2000.

[18] M.Farber, Homological algebra of Novikov-Shubin invariants and Morse inequalities. (English. English summary) Geom. Funct. Anal. 6 (1996), no. 4, 628-665.

[19] F.T.Farrell and W.C.Hsiang, Manifolds with $\pi_{1}=Z^{k}$. Manifolds-Amsterdam 1970 (Proc. Nuffic Summer School), pp. 36-43. Lecture Notes in Math., Vol. 197, Springer, Berlin, 1971.

[20] S.Ferry and S.Weinberger, A coarse approach to the Novikov conjecture. Novikov conjectures, index theorems and rigidity, Vol. 1 (Oberwolfach, 1993), 147-163, London Math. Soc. Lecture Note Ser., 226, Cambridge Univ. Press, Cambridge, 1995..

[21] M.Goresky and R.MacPherson, Intersection homology theory. Topology 19 (1980), no. 2, 135-162. Part II. Invent. Math. 72 (1983), no. 1, 77-129.

[22] P.Griffiths and J.Harris, Principles of algebraic geometry, Pure and Applied Mathematics. Wiley-Interscience, John Wiley and Sons, New York, 1978

[23] M.Gromov, Kaehler hyperbolicity and $L^{2}$-Hodge theory. J. Differential Geom. 33 (1991), no. $1,263-292$.

[24] M.Gromov, and B.Lawson, Positive scalar curvature and the Dirac operator on complete Riemannian manifolds. Inst. Hautes Études Sci. Publ. Math. No. 58 (1983), 83-196 (1984)

[25] S.Ferry, A.Ranicki, and J.Rosenberg, editors, Novikov conjectures, index theorems and rigidity. Vols. 1 and 2. Including papers from the conference held at the Mathematisches Forschungsinstitut Oberwolfach, Oberwolfach, September 6-10, 1993. Edited by Steven C. Ferry, Andrew Ranicki and Jonathan Rosenberg. London Mathematical Society Lecture Note Series, 226. Cambridge University Press, Cambridge, 1995.

[26] N.Higson and J.Roe, Analytic K-homology. Oxford Mathematical Monographs. Oxford Science Publications. Oxford University Press, Oxford, 2000.

[27] F.Hirzebruch, Topological methods in algebraic geometry. Third enlarged edition. New appendix and translation from the second German edition by R. L. E. Schwarzenberger, with an additional section by A. Borel. Die Grundlehren der Mathematischen Wissenschaften, Band 131 Springer-Verlag New York, Inc., New York 1966

[28] N.Hitchin, Harmonic spinors. Advances in Math. 14 (1974), 1-55.

[29] P.Kahn, Characteristic numbers and oriented homotopy type. Topology 3 1965 81-95.

[30] M.Kervaire and J.Milnor, Bernoulli numbers, homotopy groups, and a theorem of Rohlin. 1960 Proc. Internat. Congress Math. 1958 pp. 454-458 Cambridge Univ. Press, New York

[31] J. Kazdan, F. W. Warner, Existence and conformal deformation of metrics with prescribed Gaussian and scalar curvatures, Ann. of Math. (2) 101 (1975), 317-331.

[32] B.Lawson and S.T.Yau, Scalar curvature, non-abelian group actions, and the degree of symmetry of exotic spheres. Comment. Math. Helv. 49 (1974), 232-244.

[33] G.Lusztig, Novikov's higher signature and families of elliptic operators. J. Differential Geometry 7 (1972), 229-256.

[34] W.Lueck, $L^{2}$-invariants: theory and applications to geometry and $K$-theory. Ergebnisse der Mathematik und ihrer Grenzgebiete. 3. Folge. A Series of Modern Surveys in Mathematics [Results in Mathematics and Related Areas. 3rd Series. A Series of Modern Surveys in Mathematics], 44. Springer-Verlag, Berlin, 2002.

[35] J. Milnor, Whitehead torsion. Bull. Amer. Math. Soc. 721966 358-426.

[36] S.Novikov, Algebraic construction and properties of Hermitian analogs of $K$-theory over rings with involution from the viewpoint of Hamiltonian formalism. Applications to differential topology and the theory of characteristic classes. I. II. Math. USSR-Izv. 4 (1970), 257-292; ibid. 4 (1970), 479-505; 
[37] J.Rosenberg, An analogue of the Novikov Conjecture in complex algebraic geometry. math.AG/0509526

[38] J. Rosenberg, $C^{*}$-algebras, positive scalar curvature, and the Novikov conjecture. Inst. Hautes Études Sci. Publ. Math. No. 58 (1983), 197-212 (1984).

[39] — and S.Weinberger, Higher $G$-signatures for Lipschitz manifolds. K-Theory 7 (1993), no. 2, 101-132.

[40] J.Roe, An index theorem on open manifolds. I, II. J. Differential Geom. 27 (1988), no. 1, $87-113,115-136$

[41] L.Siebenmann, Approximating cellular maps by homeomorphisms. Topology 11 (1972), 271-294

[42] J.P.Serre, Homologie singulière des espaces fibrés. Applications. (French) Ann. of Math. (2) 54, (1951). 425-505.

[43] M.Stern, Index theory for certain complete Kaehler manifolds. J. Differential Geom. 37 (1993), no. 3, 467-503.

[44] S.Stolz, Positive scalar curvature metrics - existence and classification questions. Proceedings of the International Congress of Mathematicians, Vol. 1, 2 (Zrich, 1994), 625-636, Birkhuser, Basel, 1995.

[45] J. Schuermann, S. Yokura, A survey of characteristic classes of singular spaces, preprint AG/0511175

[46] R.Thom, Quelques propriétés globales des variétés différentiables. (French) Comment. Math. Helv. 28, (1954). 17-86.

[47] Totaro, B., Chern numbers for singular varieties and elliptic homology, Ann. of Math. (2), $151,(2000)$, no. $2,757-791$.

[48] S.Weinberger Aspects of the Novikov conjecture. Geometric and topological invariants of elliptic operators (Brunswick, ME, 1988), 281-297, Contemp. Math., 105, Amer. Math. Soc., Providence, RI, 1990

[49] S. Weinberger, Group actions and higher signatures.I, Proc. Nat. Acad. Sci. U.S.A. 82 (1985), no. 5, 1297-1298. II. Comm. Pure Appl. Math. 40 (1987), no. 2, 179-187.

[50] S. Weinberger, Class numbers, the Novikov conjecture, and transformation groups. Topology 27 (1988), no. 3, 353-365.

Jonathan Block

Department of Mathematics

University of Pennsylvania

Philadelphia, PA, USA

E-mail: blockj@math.upenn.edu

Shmuel Weinberger

Department of Mathematics

University of Chicago

Chicago, IL, USA

E-mail: shmuel@math.uchicago.edu

SW currently visiting

Courant Institute of the Mathematical Sciences

New York, NY, USA 